\title{
THE
}

UNIVERSITY

University of Rhode Island

OF RHODE ISLAND

DigitalCommons@URI

1987

\section{Toward a Monte Carlo Theory of Quantum Dynamics}

Jimmie D. Doll

R. D. Coalson

David L. Freeman

University of Rhode Island, dfreeman@uri.edu

Follow this and additional works at: https://digitalcommons.uri.edu/chm_facpubs

Terms of Use

All rights reserved under copyright.

\section{Citation/Publisher Attribution}

Doll, J. D., Coalson, R. D., \& Freeman, D. L. (1987). Toward a Monte Carlo Theory of Quantum Dynamics. Journal of Chemical Physics, 87(3), 1641-1647. doi: 10.1063/1.453226

Available at: http://dx.doi.org/10.1063/1.453226

This Article is brought to you for free and open access by the Chemistry at DigitalCommons@URI. It has been accepted for inclusion in Chemistry Faculty Publications by an authorized administrator of DigitalCommons@URI. For more information, please contact digitalcommons-group@uri.edu. 


\title{
Toward a Monte Carlo theory of quantum dynamicsa)
}

\author{
J. D. Doll \\ Los Alamos National Laboratory, Los Alamos, New Mexico 87545 \\ R. D. Coalson \\ Department of Chemistry, University of Pittsburgh, Pittsburgh, Pennsylvania 15260 \\ D. L. Freeman \\ Department of Chemistry, University of Rhode Island, Kingston, Rhode Island 02881
}

(Received 4 March 1987; accepted 3 April 1987)

\begin{abstract}
We consider in the present paper an extension of numerical path integral methods for use in computing finite temperature time correlation functions. We demonstrate that coordinate rotation techniques extend appreciably the time domain over which Monte Carlo methods are of use in the construction of such correlation functions.
\end{abstract}

\section{INTRODUCTION}

An important recent development in the theory of many-body systems has been the emergence of a variety of Monte Carlo based methods suitable for the study of problems in equilibrium quantum mechanics. ${ }^{1,2}$ These methods are proving valuable for studies of both ground state and finite temperature equilibrium systems. The developments and associated applications in this rapidly growing area are discussed elsewhere. ${ }^{1-4}$

There are well-known parallels between the formal structure of equilibrium and time-dependent quantum mechanics: the former involves density matrix elements at a real temperature while the latter involves density matrix elements at a complex temperature. Numerical difficulties associated with the appearance of complex exponentials in the dynamics problem as opposed to simple decaying terms in the equilibrium case, however, have discouraged a parallel development of Monte Carlo approaches to quantum dynamics. Some progress has been made for the restricted problem of generating short-time dynamical information, a situation where the numerical problems are less severe. For this case analytic continuation methods ${ }^{5,6}$ and direct Monte Carlo techniques ${ }^{7,8}$ have been explored. Although not a complete solution, short-time information by itself is often quite useful in problems of spectroscopy and kinetics. Basis set methods ${ }^{9,10}$ are of use in problems where the associated dimensionality does not prove overwhelming.

The present paper reports a number of related developments that suggest that the range of applicability of Monte Carlo methods in quantum dynamics is larger than previously suspected. Although it is too early to see clearly the full scope of possible future work, it appears now that a number of important problems in quantum dynamics can be pursued using methods closely related to current equilibrium approaches. After a discussion of quantum-mechanical time correlation functions in Sec. II A, we indicate in Sec. II B how slight generalizations of equilibrium quantum Monte Carlo methods can be used to compute the complex temperature density matrix elements required to describe quan-

\footnotetext{
This manuscript reports independent work and was submitted essentially simultaneously with the following manuscript.
}

tum dynamics. Several numerical examples are presented in Sec. III.

\section{THEORY}

In this section we consider the methods aimed at the construction of quantum-mechanical time correlation functions. In particular, we show that it is possible to extend equilibrium Monte Carlo path integral approaches for use in dynamical problems. Several related equilibrium formulations have been presented previously and are reviewed elsewhere. ${ }^{2}$ For simplicity, we restrict ourselves in the following to a one-dimensional notation with the understanding that the formal extension to more dimensions is straightforward. We also note that the present developments are readily adapted for use in conjunction with influence functional methods which can further simplify problems having a natural "primary system plus bath" structure.

\section{A. Time correlation functions}

We begin by considering the structure of a typical quantum-mechanical time correlation function. As discussed elsewhere, ${ }^{5,11}$ it is convenient to organize the calculation of time correlation functions in a manner that avoids the construction of pure propagators. Thus, rather than studying correlation functions of the type

$$
\begin{aligned}
C_{A B}(t)= & \operatorname{tr}[\exp (-\beta H) A \exp (i H t / \hbar) B \\
& \times \exp (-i H t / \hbar)] / \operatorname{tr} \exp (-\beta H),
\end{aligned}
$$

it is convenient to study related correlation functions

$$
\begin{aligned}
G_{\mathrm{AB}}(t)= & \operatorname{tr}\left[A \exp \left(-\beta_{c}^{*} H\right) B \exp \left(-\beta_{c} H\right)\right] \\
& \operatorname{tr} \exp (-\beta H),
\end{aligned}
$$

where $\beta_{c}$ is given by

$$
\beta_{c}=\beta / 2+i t / \hbar \text {. }
$$

It is easy to show that the Fourier transforms of $C_{A B}(t)$ and $G_{A B}(t)$ are related by

$$
G_{A B}(\omega)=\exp (-\beta \hbar \omega / 2) C_{A B}(\omega),
$$

and thus that the two functions have the same information content. However, $G_{A B}(t)$ is the more convenient function for numerical study since the propagator factors are always 
paired with a corresponding Boltzmann-like term.

The formal structure of $G_{A B}(t)$ suggests a particular direction with respect to numerical evaluation. Expanding Eq. (2.2) in a coordinate representation gives, assuming that $A$ and $B$ are operators that depend only on position,

$$
\begin{aligned}
G_{\mathrm{AB}}(t)= & \int d x d x^{\prime}\left|\left\langle x^{\prime}\left|e^{-\beta_{\mathrm{c}} H}\right| x\right\rangle\right|^{2} A(x) B\left(x^{\prime}\right) / \\
& \int d x d x^{\prime}\left|\left\langle x^{\prime}\left|e^{-\beta_{c} H}\right| x\right\rangle\right|^{2},
\end{aligned}
$$

where we have used the fact that the partition function normalization can be written as

$$
\begin{aligned}
\int d x & \langle x|\exp (-\beta H)| x\rangle \\
= & \int d x d x^{\prime}\left\langle x\left|\exp \left(-\beta_{c}^{*} H\right)\right| x^{\prime}\right\rangle\left\langle x^{\prime}\left|\exp \left(-\beta_{c} H\right)\right| x\right\rangle .
\end{aligned}
$$

Equation (2.5) gives the time correlation function $G_{A B}(t)$ as an average over a probability distribution, an average reminiscent of those that arise in equilibrium calculations. Here, however, the distribution is not a simple equilibrium factor, but rather a temperature and time-dependent probability distribution that properly expresses the dynamical correlation of the coordinates $x$ and $x^{\prime}$.

Equation (2.5) serves to focus our attention on complex temperature density matrix elements, $\left\langle x^{\prime}\left|\exp \left(-\beta_{c} H\right)\right| x\right\rangle$. Were we in a position to compute such elements, $G_{A B}(t)$ could be evaluated by Monte Carlo methods ${ }^{1,2,12}$ in much the same way that one evaluates analogous equilibrium averages. The obvious difficulty in this approach is that our ability to compute the required complex temperature density matrix elements is, at present, limited. It has generally been felt that the primary method by which we approach corresponding equilibrium problems, numerical path integral techniques, are poorly suited to the present task except at relatively short times (i.e., times small relative to $\beta h$ ).

We develop in the following section relatively simple extensions of equilibrium Monte Carlo path-integral methods that appear to overcome the difficulties traditionally associated the calculation of the complex temperature density matrix elements of the type required by Eq. (2.5). Anticipating that such methods are in fact feasible for the calculation of the required density matrix elements, we consider for the moment the problems posed by the Monte Carlo evaluation of Eq. (2.5). As written, the Monte Carlo evaluation of Eq. (2.5) requires that we sample from a probability density that is not available analytically, but rather is itself produced by a statistical process with noise. Kennedy and Kuti have considered this type of problem recently and have outlined new importance sampling methods, ${ }^{13}$ methods better able to cope with such noise than the customary Metropolis approach. ${ }^{12}$ Alternatively, we may anticipate evaluating Eq. (2.5) using either an analytically available reference probability density or a density derived from the numerical pathintegral representation of the complex temperature density matrix elements.

\section{B. Density matrix evaluation}

We consider in this section the problem of devising a practical means for estimating the density matrix elements discussed above. Since our approach will be based on extensions of equilibrium Monte Carlo path-integral methods, we briefly review these techniques to help define notation and to set the stage for further developments. As developed by Feynman ${ }^{14}$ the quantum mechanical density matrix element

$$
\rho\left(x^{\prime}, x, \beta\right)=\left\langle x^{\prime}|\exp (-\beta H)| x\right\rangle
$$

can be written in a path-integral form, giving

$$
\begin{aligned}
\rho\left(x^{\prime}, x, \beta\right)= & \int \mathscr{D} x(s) \\
& \times \exp \left(-\int_{0}^{\beta \hbar} d s\left\{\left(m \dot{x}^{2} / 2+V[x(s)]\right) / \hbar\right\}\right) .
\end{aligned}
$$

The functional integration in Eq. (2.8) corresponds to the sum over all possible paths connecting $x$ and $x^{\prime}$ satisfying the boundary conditions

$$
\begin{aligned}
& x(s=0)=x, \\
& x(s=\beta \hbar)=x^{\prime} .
\end{aligned}
$$

It is convenient to decompose the paths in Eq. (2.8) into a reference path, chosen to satisfy the boundary conditions in Eq. (2.9), and a "fluctuation" about the reference path. Since the deviations from the reference path vanish at the endpoints (by design), they can be written in a Fourier sine series. ${ }^{14}$ Using this expansion and choosing a "free-particle" reference path gives

$$
x(u)=x+\left(x^{\prime}-x\right) u+\sum_{k=1}^{\infty} a_{k} \sin (k \pi u),
$$

where the "time" variable, $u=s / \beta \hbar$, ranges over the interval $(0,1)$. The quantum mechanical paths are labeled by the Fourier coefficients $a_{k}$ and the sum over all paths in Eq. (2.8) can thus be reduced to an integral over all Fourier coefficients. It is easy to generalize the above result to utilize other types of reference paths such as the harmonic oscillator. Anticipating a Monte Carlo evaluation, we consider not $\rho$ itself but the ratio of the density matrix element at $\left(x^{\prime}, x\right)$ and at a temperature $\beta=1 / k_{B} T$ to its free-particle counterpart, $\rho_{f p}$. Using Eq. (2.10) it is easy to show ${ }^{15}$ that this ratio can be written (suppressing the explicit coordinate dependence for simplicity) as

$$
\begin{aligned}
\rho / \rho_{f p}= & \int d \mathrm{a} \exp \left(-\sum_{k=1}^{\infty} a_{k}^{2} / 2 \sigma_{k}^{2}-\beta\langle V\rangle\right) / \\
& \int d \mathbf{a} \exp \left(-\sum_{k=1}^{\infty} a_{k}^{2} / 2 \sigma_{k}^{2}\right) .
\end{aligned}
$$

The two terms in the exponent of the numerator of Eq. (2.11) originate from the kinetic and potential energy pieces of the "action" integral in Eq. (2.8). Analytic evaluation of the kinetic energy term generates the Gaussians in Eq. (2.11) whose widths, $\sigma_{k}^{2}$, are given by

$$
\sigma_{k}^{2}=2 \beta \hbar^{2} /\left(m \pi^{2} k^{2}\right)
$$

These second moments have a de Broglie-like dependence on mass and temperature and decreases with increasing $k$. The 
potential energy term corresponds to the average of $V[x(u)]$ over the particular path specified by the $a_{k}$ variables. Explicitly,

$$
\langle V\rangle=\int_{0}^{1} V[x(u)] d u .
$$

Unlike the average over the kinetic energy, the average over the potential energy is not generally available analytically and must be evaluated through the use of numerical quadrature methods. Since this integral is one dimensional even for general problems, however, this numerical task is not difficult. In the present work we have used a trapezoidal quadrature scheme, writing the average of the potential as

where

$$
\langle V\rangle=\sum_{n=1}^{N} V\left[x\left(u_{n}\right)\right] / N,
$$

$$
u_{n}=n / N \text {. }
$$

The Appendix indicates how standard Fourier transform methods are of assistance in the evaluation of Eq. (2.13b).

The formally infinite Fourier expansion of the quantum mechanical paths will, in practice, be truncated at some finite upper index. The fact that the second moments of the free-particle Gaussians decay with increasing Fourier index implies that such a truncation will be practical for typical interaction potentials. In the simplest implementation of the Fourier approach, contributions of the higher-order Fourier expansion coefficients are completely discarded. It is possible, however, to include such contributions (approximately) through the method of partial averaging, ${ }^{16,17}$ a relatively simple extension of the basic Fourier method. In this approach the effects of the high-order Fourier coefficients are included through a mass and temperature dependent effective potential. The prescription for the construction of this effective potential and examples of its use are discussed elsewhere. ${ }^{16,17}$

Equations (2.10)-(2.12) are appropriate for complex as well as real values of $\beta^{8,17}$ In particular, if we have a complex value of $\beta, \beta_{c}$, given by

$$
\beta_{c}=\beta+i t / \hbar \text {, }
$$

then Eqs. (2.10) - (2.12) provide us with a device for generating (formally, at least) the corresponding complex temperature density matrix elements. As discussed in the preceding section, the motivation for examining such matrix elements is that they arise naturally in the study of finite temperature time correlation functions.

Before proceeding it is useful to illustrate the difficulties inherent in the direct Monte Carlo calculation of complex temperature density matrix elements, difficulties that have effectively discouraged previous efforts in this direction. Defining $s_{k}^{2}$ as

$$
s_{k}^{2}=2\left|\beta_{c}\right|^{2} \hbar^{2} /\left(m \pi^{2} k^{2} \beta\right),
$$

we can rewrite Eq. (2.11) as

$$
\begin{aligned}
\rho / \rho_{f p}= & \int d \mathbf{a} \exp \left[-\left(\sum_{k=1}^{\infty} a_{k}^{2} / 2 s_{k}^{2}+\beta\langle V\rangle\right)\right. \\
& \left.+(i t / \beta \hbar)\left(\sum_{k=1}^{\infty} a_{k}^{2} / 2 s_{k}^{2}-\beta\langle V\rangle\right)\right] / \\
& \int d \mathbf{a} \exp \left(-\sum_{k=1}^{\infty} a_{k}^{2} / 2 s_{k}^{2}+(i t / \beta \hbar) \sum_{k=1}^{\infty} a_{k}^{2} / 2 s_{k}^{2}\right) .
\end{aligned}
$$

From Eqs. (2.15) and (2.16) we can see two related problems exist. First, comparing Eqs. (2.15) and (2.12), we see that in the complex temperature case the parameter $\left|\beta_{c}\right|^{2} / \beta$ plays the role of an effective temperature. Longer real times in a dynamics problem are thus analogous to lower temperatures in the equilibrium case. This implies that at longer real times more variables will be required in the expansions of the quantum mechanical paths. The low effective temperature, a technical detail in equilibrium problems, is more troublesome in the case of dynamics for reasons that will soon become apparent. A second problem centers on the integrands in the numerator and denominator of Eq. (2.16). The complex exponentials in each oscillate for nonzero values of $t$, with the ratio $t / \beta \hbar$ being a natural parameter describing the time scale for the onset of rapid oscillations. The rapid oscillations at long times have led in the past to a general pessimism concerning the possibility of developing Monte Carlo numerical procedures for the evaluation of path-integral averages in quantum dynamics for times beyond roughly $\beta \hbar$.

We begin our development with an observation. A direct calculation of Eq. (2.16) via Monte Carlo methods would typically proceed by first splitting up the Gaussian kinetic energy terms in the numerator and denominator of Eq. (2.16) into real and imaginary parts. Equation (2.16) would thus be expressed as the ratio of two separate averages, each computed using the real portion of the kinetic energy term as a weight function. We note, however, that so constructed the denominator would equal $(1-i t / \beta \hbar)^{-k_{\max } / 2}$, where $k_{\max }$ is the number of terms included in the Fourier path expansion. As $t$ (and thus $k_{\max }$ ) becomes large, this vanishing contribution would have to be cancelled by the numerator in order to achieve a finite result. Although it is easy to see that this would occur formally, achieving the cancellation numerically would generally be quite difficult. We conclude from this observation that if we are to succeed in developing Monte Carlo methods for the calculation of $\rho$, then we must generally avoid transforming Eq. (2.16) into two separate Monte Carlo problems.

To proceed we return to our original path-integral expression, Eq. (2.11). It is convenient to separate the Fourier coefficients that appear in the path-integral into three broad catagories based on the magnitude of their free particle widths, $\sigma_{k}$, given by Eq. (2.12). For $k$ beyond a particular value, $k_{\max }$, the $\sigma_{k}$ value will be sufficiently small in magnitude that the associated $a_{k}$ variables will behave in an essentially free-particle-like fashion. This suggests that we treat the high-order coefficients by partial averaging. ${ }^{16,17}$ Rather than simply truncating the expansions at $k=k_{\max }$ and completely ignoring contributions from higher-order terms, we utilize a low-order cumulant treatment of the averages, replacing "the average of the exponential by the exponential of the average." The effects of the high-order Fourier coefficients thus appear through a (complex) temperature and mass dependent potential given in terms of the original potential, $V(x)$, by

$$
\begin{aligned}
V_{p a}(x, u)= & \int d y \exp \left[-y^{2} / 2 \sigma^{2}(u)\right] V(x+y) / \\
& \int d y \exp \left[-y^{2} / 2 \sigma^{2}(u)\right],
\end{aligned}
$$


where $\sigma^{2}(u)$ is given by

$$
\begin{aligned}
\sigma^{2}(u)= & \left(\beta_{c} \hbar^{2} / m\right)[u(1-u) \\
& \left.-\left(2 / \pi^{2}\right) \sum_{k=1}^{k_{\max }} \sigma_{k}^{2} \sin ^{2}(k \pi u)\right] .
\end{aligned}
$$

This procedure is closely related in spirit to the FeynmanHibbs effective potential construction. ${ }^{18}$ Here, however, the averaging process is designed to include only the high-order terms rather than the entire quantum correction. The value of $k_{\max }$ that is required for any particular problem can be anticipated by comparing the magnitudes of the partial averaging width and the length scale for variation of the interaction potential.

The effectiveness of partial averaging has been discussed in detail elsewhere. ${ }^{16,17} \mathrm{~A}$ rough indication of its utility is illustrated in Table $\mathrm{I}$. We list there the numerical values, obtained analytically, for the ratio of the $x^{\prime}=x=0$ density matrix element to its free-particle value with and without partial averaging for the case of a parabolic barrier, $V(x)=-m \omega^{2} x^{2} / 2$. This ratio is given as a function of the number of explicit Fourier coefficients retained in Eq. (2.11) for the particular case of $\beta \hbar \omega=1$ and $t=0$. We see from Table I that partial averaging substantially reduces the number of explicit Fourier coefficients required. Similar reductions have been observed on a variety of equilibrium and dynamical problems. Although we will not explicitly indicate it in our subsequent notation, we will generally assume in what follows that the high-order coefficients have been included using partial averaging.

We now turn to the central issue in the current problem and consider the integrals over the remaining Fourier coefficients in Eq. (2.11). A convenient way to avoid transforming Eq. (2.11) into two separate problems is to introduce a coordinate rotation chosen to render the natural weight function, in this case the Gaussians from the kinetic energy term, entirely real. Using a coordinate rotation defined by

$$
\alpha_{k}^{2}=a_{k}^{2} / \sigma_{k}^{2} \text {, }
$$

gives in place of Eq. (2.11) the ratio

$$
\begin{aligned}
\rho / \rho_{f p}= & \int d \alpha \exp \left(-\sum_{k=1}^{k_{\max }} \alpha_{k}^{2} / 2-\beta_{c}\langle V\rangle\right) / \\
& \int d \alpha \exp \left(-\sum_{k=1}^{k_{\max }} \alpha_{k}^{2} / 2\right) .
\end{aligned}
$$

TABLE I. Listed are the ratios of Fourier density matrix elements to their free-particle counterparts for a $\beta \hbar \omega=1$ parabolic barrier $\left[V(x)=-m \omega^{2} x^{2} / 2\right]$. The results shown are with (PA) and without (FPI) partial averaging and are shown as a function of the number of explicit Fourier coefficients retained in the expansion of the quantum paths, $k_{\max }$ [cf. Eq. (2)]. The $k_{\max }=0$ FPI result is, by construction, the freeparticle value. The exact ratio is given analytically by $(\beta \hbar \omega / \sin \beta \hbar \omega)^{1 / 2}$ and is $\mathbf{1 . 4 8 3}$ for the present case.

\begin{tabular}{rll}
\hline \hline$k_{\max }$ & FPI & PA \\
\hline 0 & 1.000 & 1.396 \\
1 & 1.297 & 1.478 \\
2 & 1.368 & 1.482 \\
3 & 1.400 & 1.483 \\
20 & 1.469 & 1.483 \\
100 & 1.480 & 1.483 \\
\hline
\end{tabular}

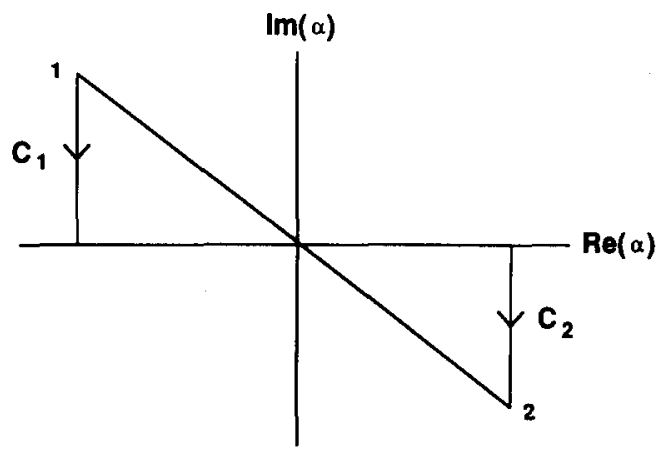

FIG. 1. The integration contour for the $\alpha_{k}$ integrals in Eq. (2.20). The original integral runs along the line from point 1 to point 2 in the figure. The distorted contour includes contours $C_{1}$ and $C_{2}$ as well as the real- $\alpha$ axis.

The paths in Eq. (2.20) are specified by

$$
x(u)=x+\left(x^{\prime}-x\right) u+\sum_{k=1}^{k_{\max }} \alpha_{k} \sigma_{k} \sin k \pi u,
$$

and the $\alpha_{k}$ integrations in Eq. (2.20) are now along a line in the complex $\alpha$ plane from $-\infty / \sigma_{k}$ to $\infty / \sigma_{k}$. (See Fig. 1.) Since all $\sigma_{k}$ values are proportional to $\beta_{c}$, the rotation angle is the same for all Fourier coefficients and the Fourier sum in Eq. (2.21) is distributed along a single line in the complex $\alpha$ plane. Equation (2.20) is formally equivalent to Eq. (2.11). When $\exp \left(-\Sigma \alpha_{k}^{2} / 2-\beta_{c}\langle V\rangle\right)$ is analytic within the regions bounded by the original contour, the real axis and contours $C_{1}$ and $C_{2}$, the integrals in Eq. (2.20) can be evaluated along the distorted contours made up of $C_{1}, C_{2}$, and the real axis. Furthermore, if $\exp \left(-\Sigma \alpha_{k}^{2} / 2-\beta_{c}(V\rangle\right)$ vanishes along $C_{1}$ and $C_{2}$ we have

$$
\begin{aligned}
\rho / \rho_{f p}= & \int_{-\infty}^{\infty} d \alpha \exp \left(-\sum_{k=1}^{k_{\max }} \alpha_{k}^{2} / 2-\beta_{c}\langle V\rangle\right) / \\
& \int_{-\infty}^{\infty} d \alpha \exp \left(-\sum_{k=1}^{k_{\max }} \alpha_{k}^{2} / 2\right) .
\end{aligned}
$$

Equation (2.22) expresses the desired density matrix element as a single average of the type discussed elsewhere. ${ }^{19}$ It remains to demonstrate that the numerical evaluation of $\mathrm{Eq}$. (2.22) is feasible for interesting physical systems.

Before presenting numerical examples, we consider the application of the above results to a model problem where we can explore the various steps analytically. We consider first the problem of computing the ratio of the density matrix element to its free-particle value for the $x^{\prime}=x=0$ parabolic barrier, $V(x)=-m \omega^{2} x^{2} / 2$. It is straightforward to show that for this problem Eq. (2.22) reduces to

$$
\begin{aligned}
& \rho / \rho_{f p} \\
& =\int_{-\infty}^{\infty} d \alpha \exp \left\{-\sum_{k=1}^{k_{\max }} \alpha_{k}^{2}\left[\left(1 / 2-\beta_{c}^{2} \hbar^{2} \omega^{2} /\left(2 \pi^{2} k^{2}\right)\right]\right\} /\right. \\
& \int_{-\infty}^{\infty} d \alpha \exp \left(-\sum_{k=1}^{k_{\max }} \alpha_{k}^{2} / 2\right) .
\end{aligned}
$$

Convergence of the $\alpha_{k}$ th integral in the numerator of Eq. (2.23) is assured if

$$
\operatorname{Re}\left[1 / 2-\beta_{c}^{2} \hbar^{2} \omega^{2} /\left(2 \pi^{2} k^{2}\right)\right]>0,
$$


which implies that $t$ must satisfy

$$
t^{2}>\beta^{2} \hbar^{2}-\pi^{2} k^{2} / \omega^{2}
$$

For a fixed value of $\beta$ there thus exists a time, given by Eq. (2.25), beyond which rotation is appropriate for the $\alpha_{k}$ th integration. For $\beta \hbar \omega<\pi$ the coordinate rotation procedure is formally correct for all coefficients for all times. For a general value of $\beta \hbar$, rotation will be appropriate for the higher coefficients and inappropriate for the lower ones, implying that some alternate method of evaluation for these loworder terms will be required. In contrast to the direct formulation of the dynamics problem, Eq. (2.16), which ultimately led to a ratio of two vanishing Monte Carlo averages, direct Monte Carlo integration over these few variables proves to be feasible. Methods designed specifically to cope with such problems have been discussed elsewhere. ${ }^{19}$ Our analysis suggests that the calculation of complex temperature density matrix elements for barrier type problems should be relatively easy, even for large real times. Repeating the above analysis for an ordinary harmonic oscillator, $V(x)=m \omega^{2} x^{2} / 2$, leads to the conclusion that coordinate rotation for the $\alpha_{k}$ th variable is valid for times satisfying

$$
t<(\pi k / \omega) V\left[1+(\beta \hbar \omega / \pi k)^{2}\right] \text {. }
$$

For oscillatory problems we thus expect that coordinate rotation using a free-particle reference will work well for all degrees of freedom for times less than roughly half a vibrational period and then to fail for successive Fourier variables at the rate of approximately one variable per half vibrational period. As above, we expect that direct Monte Carlo methods or their extensions ${ }^{19}$ will be useful for these integrations where rotation fails. For bound degrees of freedom an oscillator reference system, rather than the free-particle form discussed here, may prove advantageous.

We expect remnants of the above structure to survive for anharmonic potentials and we also expect that a combination of coordinate rotation, direct Monte Carlo and stationary phase Monte Carlo methods ${ }^{19}$ to be of use in the construction of the relevant averages.

In summary, we have presented an approach aimed at the calculation of complex temperature density matrix elements based on a combination of coordinate rotation and direct Monte Carlo methods. By partitioning path-integral variables into classes and treating each of these classes in a natural way, we have appreciably reduced the difficulty traditionally associated with the development of a quantum Monte Carlo dynamics.

\section{NUMERICAL EXAMPLES}

It is useful to begin our discussion with a simple oscillator example outlined analytically in the previous section. In what follows we will artificially evaluate the oscillator density matrix elements numerically, using a free-particle reference system and taking no special advantage of the quadratic nature of the potential energy. Both the $\alpha_{k}$ integrations in Eq. (2.22) as well as the one-dimensional quadrature of the time average over the potential energy function, Eq. (2.13), were evaluated numerically. Specifically, the $\alpha_{k}$ integrations were performed by ordinary Monte Carlo methods and the $u$ integrations were evaluated by trapezoidal quadrature (see the Appendix ).

Figure 2 displays the real part of the $x^{\prime}=x=0$ parabolic barrier $\left[V(x)=-m \omega^{2} x^{2} / 2\right]$ density matrix element as a function of time. For these results the complex temperature was $\beta_{c}=\beta / 2+i t / \hbar$ and other parameters were $m=m_{H}, \omega / k_{B}=1000 \mathrm{~K}$, and $T=500 \mathrm{~K}$ [a temperature for which Eq. (2.25) is satisfied for all values of $t$ and $k$ ]. For reference, $\beta \hbar / 2=316$ a.u. at this temperature and $2 \pi /$ $\omega=1984$ a.u. We see first from Fig. 2 that with coordinate rotation that it is quite easy to compute density matrix elements for the barrier problem for values of $t$ large compared with $\beta \hbar / 2$. For comparison, the error estimate for the $t=2000$ a.u. result obtained without rotation methods using the same number of Monte Carlo points and Fourier coefficients is of the order of $10^{18}$ times larger than when rotation is used, implying that for this particular circumstance that rotation methods represent approximately a $10^{36}$ gain in computational efficiency relative to the direct Monte Carlo approach. The gain in computational efficiency for the rotation method relative to the direct Monte Carlo approach is substantially larger at longer real times. Similar results, albeit with slightly larger statistical errors for the same numerical effort, can be obtained for the density matrix elements for the ordinary harmonic oscillator.

We now turn to a discussion of anharmonic systems and consider first the problem of particle passage over an anharmonic Eckart barrier,

$$
V(x)=V_{0} \operatorname{sech}^{2}(x / a) .
$$

We note that since $\operatorname{sech}(z)$ vanishes for large $|z|[\operatorname{Re}(z) \neq 0]$, the arguments leading to Eq. (2.22) are valid. A convenient way to analyze the kinetics associated with this barrier passage is through the flux autocorrelation function, ${ }^{5}$ a function expressable in terms of coordinate de-

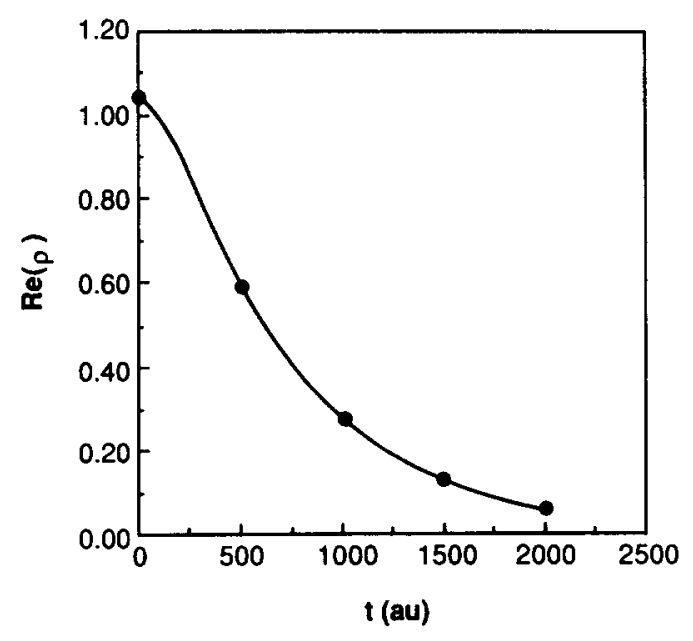

FIG. 2. Shown is the real part of the $x^{\prime}=x=0$ complex temperature $\left(\beta_{c}=\beta / 2+i t / \hbar\right)$ density matrix element for the parabolic barrier $\left(m=m_{H}, \omega / k_{B}=1000 \mathrm{~K}, T=500 \mathrm{~K}\right.$ ) as a function of time. The line corresponds to the exact result and the points are produced by a coordinate rotation Monte Carlo estimate [Eq. (2.23)] using $k_{\max }=8$ with 2000 Monte Carlo points being used in each estimate. 
rivatives of the complex temperature density matrix elements. The calculation of this correlation function via Monte Carlo methods suffices to illustrate that we cannot only compute the relevant density matrix elements for an anharmonic barrier problem, but that we can compute them with sufficient accuracy to extract physically interesting information. In order to facilitate comparison with Wyatt's RRGM calculation, ${ }^{10(c)}$ we choose as system parameters $m=m_{H}, V_{0}=2500 \mathrm{~cm}^{-1}$, and $a=1$ a.u. Physically, this corresponds to a hydrogen atom crossing a $7.15 \mathrm{kcal} / \mathrm{mol}$ barrier. Shown in Fig. 3 is the flux autocorrelation function, $C_{f}(t)$, evaluated at $x=0$ for this potential computed using the present coordinate rotation methods. ${ }^{20}$ The results in Fig. 3 are in excellent agreement with the corresponding results in Fig. 2 of Ref. 10(c). Although not plotted, values of $C_{f}(t)$ for appreciablly larger times than those shown in Fig. 3 could be easily generated for the present problem using the coordinate rotation approach. The integral of $C_{f}(t)$ from $t=0$ to infinity gives the thermal rate constant for barrier passage.

Finally, we turn to a more challenging test of the present approach and examine the dynamics of an anharmonic oscillatory degree of freedom. As an illustration that such problems can be studied, we consider the dynamics of a system with a potential of the form

$$
\begin{aligned}
V(x)= & V_{0}\left(\operatorname { e x p } \left\{-[(x+a) / \sigma]^{2} / 2\right.\right. \\
& \left.+\exp \left(-[(x-a) / \sigma]^{2} / 2\right\}\right) .
\end{aligned}
$$

This potential is plotted in Fig. 4 for the particular parameters

$$
\begin{aligned}
& V_{0}=1.139 \times 10^{-2} \text { a.u., } \\
& a=2 \text { a.u., } \\
& \sigma=1 \text { a.u. }
\end{aligned}
$$

We have chosen a potential expressed as a linear combination of Gaussians in order to simplify the partial averaging process and also since such Gaussian expansions may prove

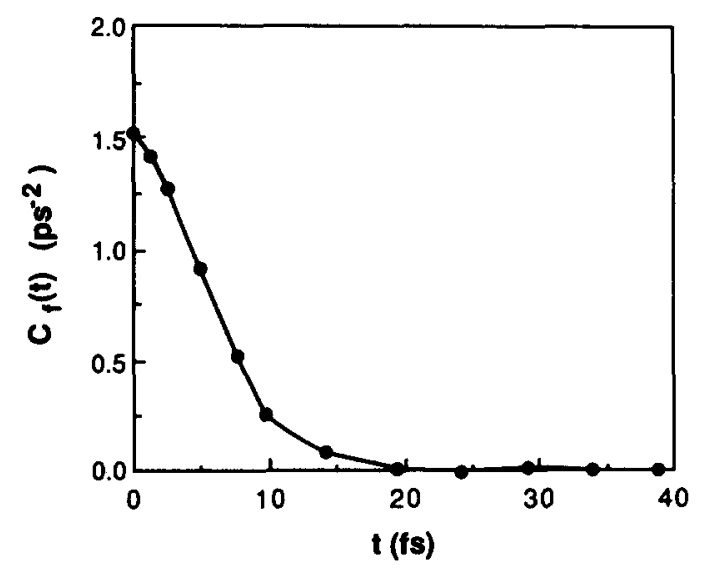

FIG. 3. Shown is the flux autocorrelation function $(x=0)$ for the potential given in Eq. (3.1). The points correspond to $k_{\max }=1$ estimates each computed using the methods of Sec. II utilizing 10000 Monte Carlo points for each estimate. The points are connected by a smooth curve as a visual aid. The results are in good agreement with those discussed in Ref. 10(c). a useful general representation for interaction potentials in conjunction with the present rotation methods. It is straightforward to compute the density matrix elements for this potential using a free-particle reference for times exceeding one vibrational period using a combination of the coordinate rotation and Monte Carlo methods outlined above. To illustrate this, we plot in Fig. 5 the flux autocorrelation function (evaluated at $x=0$ ) computed using these methods for $m=m_{H}$ and $T=500 \mathrm{~K}$. The solid line in Fig. 5 corresponds to results obtained using the NMM method ${ }^{21}(P=128)$. For reference, the relevant thermal time is $\beta \hbar / 2=7.6 \mathrm{fs}$. Several things are evident from Fig. 5. First, we see evidence of appreciable anharmonic behavior. We also see in the decay of successive maxima of $C_{f}(t)$ an indication of leakage of particles initially confined within the potential well through the Gaussian barriers. (Were we specifically interested in computing the escape of particles from this potential well, we would obviously place our flux counting surface in a barrier region rather than at the local minimum of the potential.) This example illustrates that using the present methods it is possible to compute accurately physically interesting finite temperature correlation functions for an anharmonic oscillatory degree of freedom for times well in excess of $\beta \hbar$ and beyond a single vibrational period.

\section{DISCUSSION AND SUMMARY}

We have presented here a number of related developments that would appear to broaden significantly the applicability of Quantum Monte Carlo methods with respect to the study of dynamics. Utilizing a combination of techniques centered around coordinate rotation, we have shown that it is possible to extend the time domain over which one can evaluate complex temperature density matrix elements and associated properties using Monte Carlo methods well beyond the $\beta \hbar$ value previously thought to represent an upper limit. The present results suggest that often expressed pessimism concerning the applicability of quantum Monte Carlo

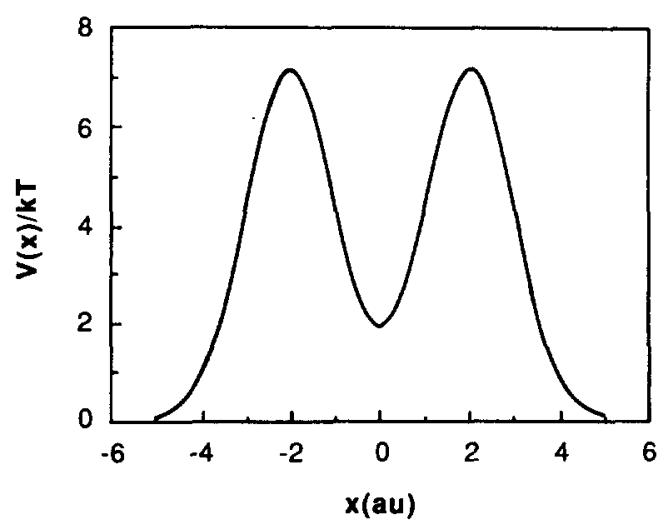

FIG. 4. The potential defined by Eqs. (3.2) $(T=500 \mathrm{~K})$. 


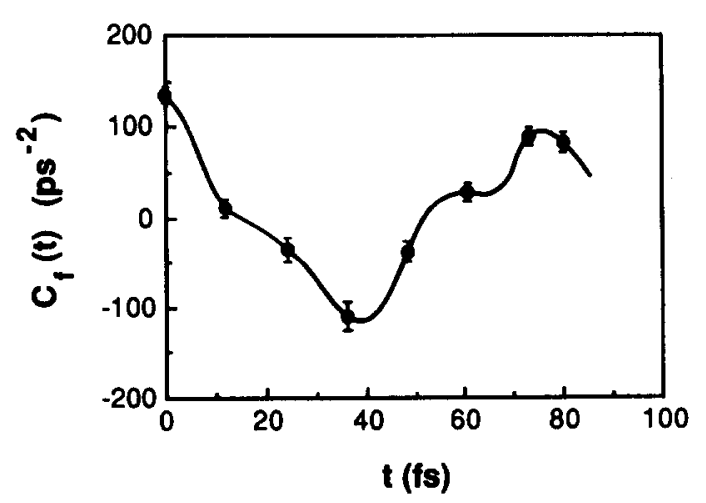

FIG. 5. The flux autocorrelation function $(x=0)$ for the double barrier potential plotted in Fig. 4. The solid line corresponds to NMM estimates $(P=128)$. The points represent calculations in which the methods of Sec. II were used to estimate the necessary complex temperature density matrix elements $\left(m=m_{H}, T=500 \mathrm{~K}, k_{\max }=16\right)$. Finite difference methods were used to compute the necessary coordinate derivatives. A common mesh size ( 0.1 a.u.) was used for both sets of results to facilitate a direct comparison. The number of Monte Carlo points used varied from $10^{4}$ for times out to $40 \mathrm{fs}$ to $10^{6}$ for the longer times. The relevant thermal time scale, $\beta \hbar / 2$, is $7.6 \mathrm{fs}$ at these temperatures.

methods to problems in dynamics was premature. The simple examples presented suggest, in fact, that the present approach will be useful for important classes of dynamics problems. We caution, however, that additional work will be required in order to establish the general limitations of the present methods.

One feature of the present development that requires further attention is the issue of the representation of empirical interaction potentials. As in semiclassical $S$-matrix theory $^{22}$ for classically forbidden processes, the present procedure probes the behavior of the potential throughout the complex plane, implying that certain representations of these interactions will likely be more convenient than others.

\section{ACKNOWLEDGMENTS}

We would like to thank H. K. McDowell and L. R. Pratt for many stimulating conversations concerning various aspects of the present work. We would also like to thank Paul Cunningham of CLS- 1 and the Chemistry and Laser Sciences Division at Los Alamos National Laboratory for their support. Work at the University of Rhode Island was supported in part by Grants from the Research Corporation and the University of Rhode Island Academic Computer Center. By mutual agreement, this work is being published in conjunction with the work of Professor W. H. Miller and collaborators at Berkeley. We acknowledge a mutual exchange of preprints prior to publication.

\section{APPENDIX}

In performing Fourier path integral Monte Carlo calculations one needs to compute rapidly quantities of the type

$$
S_{n}=\sum_{k=1}^{k_{\max }} a_{k} \sin (k \pi n / N), \quad n=1,2, \ldots, N
$$

where the $\left\{a_{k}\right\}$ values are the Fourier coefficients specifying a particular path. For typical applications we have $N \approx k_{\max }$, which means that computing all $S_{n}$ values is apparently a task of effort proportional to $N^{2}$. We note, however that the sum in Eq. (A1) is of the form of a finite sine transform of the array $\left\{a_{k}\right\}$. If the Monte Carlo sampling algorithm for the $a_{k}$ values involves making simultaneous changes in several variables, it is convenient to compute Eq. (A1) using fast sine transform methods ${ }^{24}$ to reduce the effort from order $N^{2}$ to order $N \log N$.

'For a cross section of recent work in this area, see J. Stat. Phys. 43, 7291244 (1986).

${ }^{2}$ B. J. Berne and D. Thirumalai, Annu. Rev. Phys. Chem. 37, 401 (1986). ${ }^{3}$ D. Ceperley and B. J. Alder, Science 231, 555 (1986).

4J. D. Doll and D. L. Freeman, Science 234, 1356 (1986).

${ }^{5}$ W. H. Miller, S. D. Schwartz, and J. W. Tromp, J. Chem. Phys. 79, 4889 (1983).

${ }^{6}$ D. Thirumalai and B. J. Berne, J. Chem. Phys. 81, 2512 (1984); 79, 5029 (1983).

${ }^{7}$ E. C. Behrman, G. A. Jongeward, and P. G. Wolynes, J. Chem. Phys. 79, 6277 (1983); 83, 668 (1985); E. C. Behrman and P. G. Wolynes, J. Chem. Phys. 83, 5863 (1985).

8J. D. Doll, J. Chem. Phys. 81, 3536 (1984).

${ }^{9}$ Reference 5, Sec. IV; see also N. Marki and W. H. Miller, J. Chem. Phys. 86, 1451 (1987).

${ }^{10}$ (a) A. Nauts and R. E. Wyatt, Phys. Rev. Lett. 51, 2238 (1983); (b) R. A. Friesner and R. E. Wyatt, J. Chem. Phys. 82, 1973 (1985); (c) R. E. Wyatt, Chem. Phys. Lett. 121, 301 (1985).

"D. Thirumalai and B. J. Berne, J. Chem. Phys. 81, 2512 (1984); B. J. Berne and C. D. Harp, Adv. Chem. Phys. 17, 63 (1970).

${ }^{12}$ N. Metropolis, A. W. Rosenbluth, M. N. Rosenbluth, A. H. Teller, and E. Teller, J. Chem. Phys. 21, 1087 (1953); J. P. Valleau and S. G. Whittington, in Modern Theoretical Chemistry, edited by B. J. Berne (Plenum, New York, 1977), Vol. 5, pp. 137-168.

${ }^{13}$ A. D. Kennedy and J. Kuti, Phys. Rev. Lett. 54, 2473 (1985).

${ }^{14}$ R. P. Feynman and A. H. Hibbs, Quantum Mechanics and Path Integrals (McGraw-Hill, New York, 1965).

15J. D. Doll and D. L. Freeman, J. Chem. Phys. 80, 2239 (1984); D. L. Freeman and J. D. Doll, ibid. 80, 5709 (1984); 82, 462 (1985); Adv. Chem. Phys. (in press).

${ }^{16}$ J. D. Doll, R. D. Coalson, and D. L. Freeman, Phys. Rev. Lett. 55, 1 (1985).

${ }^{17}$ R. D. Coalson, D. L. Freeman, and J. D. Doll, J. Chem. Phys. 85, 4567 (1986).

${ }^{18}$ Reference 14, Chap. (10.3).

${ }^{19} \mathrm{~J}$. D. Doll and D. L. Freeman, Adv. Chem. Phys. (in press).

${ }^{20}$ These results and the associated formalism were presented and discussed publicly at the Lasers, Molecules, and Methods Conference, Los Alamos, New Mexico, July, 1986.

${ }^{21}$ D. Thirumalai and B. J. Berne, J. Chem. Phys. 79, 5063 (1983).

${ }^{22} \mathrm{~W}$. H. Miller, Science 233, 171 (1986), and references therein.

${ }^{23}$ See, for example, W. H. Press, B. P. Flannery, S. A. Teukolsky, and W. T. Vetterling, Numerical Recipes (Cambridge University, Cambridge, 1986), Chap. 12. 
The Journal of Chemical Physics is copyrighted by the American Institute of Physics (AIP). Redistribution of journal material is subject to the AIP online journal license and/or AIP copyright. For more information, see http:/ojps.aip.org/jcpo/jcpcr/jsp Copyright of Journal of Chemical Physics is the property of American Institute of Physics and its content may not be copied or emailed to multiple sites or posted to a listserv without the copyright holder's express written permission. However, users may print, download, or email articles for individual use. 
The Journal of Chemical Physics is copyrighted by the American Institute of Physics (AIP). Redistribution of journal material is subject to the AIP online journal license and/or AIP copyright. For more information, see http://ojps.aip.org/jcpo/jcper/jsp 